\title{
The Treatment of Symptoms in Atopic Dermatitis as a Superantigen Disease
}

\author{
Sugimoto $\mathrm{K}^{*}$, Hattori $\mathrm{T}^{2}$, Kitsukawa $\mathrm{Y}^{3}$, Aotsuka $\mathrm{A}^{3}$, Wada $\mathrm{T}^{3}$, Kubosawa $\mathrm{H}^{4}$ and Ito $\mathrm{S}^{5}$ \\ ${ }^{1}$ Departments of Clinical Allergology, Neurology Clinic Tsudanuma, Japan \\ ${ }^{2}$ Departments of Neurology, Neurology Clinic Tsudanuma, Japan \\ ${ }^{3}$ Internal Medicine, Chiba Aoba Municipal Hospital, Japan \\ ${ }^{4}$ Clinical Pathology, Chiba Aoba Municipal Hospital, Japan \\ ${ }^{5}$ Department of Neurology, Chiba University Graduate School of Medicine, Japan
}

${ }^{*}$ Corresponding author: Sugimoto K, Departments of Clinical Allergology, Neurology Clinic Tsudanuma, Japan, E-mail: ka.sugimoto.chiba.jp@nifty.com

Citation: Sugimoto K, Hattori T, Kitsukawa Y, Aotsuka A, Wada T, et al. (2016) The Treatment of Symptoms in Atopic Dermatitis as a Superantigen Disease. J Microbiol Modern Tech 1(1): 105

Received Date: September 13, 2016 Accepted Date: October 24, 2016 Published Date: October 26, 2016

\begin{abstract}
Atopic dermatitis affects the skin of patients, as well as other organs, causing rash, intestinal tract and cervical spine disorder. This disease is aided by Staphylococcus aureus, which is an underlying factor producing a toxin (superantigens) to aid the onset and exacerbation of Atopic dermatitis (AD). This research proposed a treatment for the symptoms of the disease - skin rash, intestinal tract and cervical spine disorders. The treatment proposed (by the use of combined disinfectants) proofed effective for the treatment of skin rash. The researchers hope that this treatment will be adopted by health practitioners. Further study could look into the treatment of skin rash, along with intestinal tract and cervical spine disorders.
\end{abstract}

Keywords: Atopic dermatitis; Staphylococcus aureus; Superantigen; Disinfectant; IgE antibodies to SEA and SEB

\section{Introduction}

In atopic dermatitis skin barrier mechanism is destroyed, is a disease that causes chronic a variety of symptoms in atopic dermatitis to receive a lot of stimulus from there. There is a susceptibility to infection in one of the pathogenesis of atopic dermatitis [1-4]. Kobayashi, et al. reported that Staphylococcus aureus is involved in the onset and exacerbation of AD [5]. Regarding the disorder of organs other than the skin of patients with AD, Yamada, et al. of Kinki University reported colitis by biopsy, Kino, et al. of Kansai Medical University reported jejunitis of infants by ultrasonic diagnosis, and Kira, et al. of Kyushu University reported atopic myelitis [6-8]. Ito, et al. checked the neurologic findings and Magnetic resonance imaging (MRI) of patients with AD, they reported that $\mathrm{AD}$ might become a risk factor in disc degeneration [9]. From the skin in the patients of moderate or severe $\mathrm{AD}$, Staphylococcus aureus has been detected 100\%. Staphylococcus aureus produce toxins at a high frequency. Some strains produce several toxins, such as staphylococcal enterotoxin A (B, C, E), toxic shock syndrome toxin and exfoliative toxin A (B). The toxins produced by Staphylococcus aureus act as superantigen [10]. In patients with $\mathrm{AD}$ there are many reports that there is a correlation between changes in the severity of $\mathrm{AD}$ and the transition of levels of IgE antibodies to Staphylococcal Enterotoxin A (SEA) and Staphylococcal Enterotoxin A (SEB) [11-14].

\section{Methods}

In addition to the conventional treatment with topical steroids and moisturizing agents for AD patients the researchers treated Staphylococcus aureus using disinfectants, such as Isodine ${ }^{\circledast}$ solution [15-19]. As a result, short term improvements in patients' skin rash were seen. The researchers hypothesized that Staphylococcus aureus, acting as a superantigen, is the underlying cause of the disorder of many organs including the skin, in patients with AD. For the presence or absence of the patient to the organ involvement other than the skin of $\mathrm{AD}$, to those who wish to such inspection, authors carried out duodenal biopsy and or the neurological study. The toxin produced by Staphylococcus aureus was analyzed using Polymerase Chain Reaction (PCR). Also, total $\mathrm{IgE}$ and IgE antibodies to SEA and SEB during the course treatment of patients with AD were measured.

\section{Results}

Staphylococcus aureus produces many kinds of toxins. The detection rate of toxins was $80.1 \%$ from 196 Staphylococcus aureus strains [15]. Staphylococcus aureus was detected on newborn patients' skins who developed AD. In addition, the researchers discovered 
that $\mathrm{IgE}$ antibodies to SEA and SAB were positive in the serum of babies with $\mathrm{AD}$, a few months after birth. In the study during the treatments proposed for rash, IgE antibodies to SEA and SEB in patients were improving. Researchers reported duodenitis in 43 cases in duodenal biopsies of patients with $\mathrm{AD}$ of the 53 cases. Along with the improvement of the rash by Staphylococcal disinfection skin care method for $\mathrm{AD}$ that developed, of 12 patients with repeated duodenum biopsies, the duodenitis was normalized in five cases [15].

Using MRI, and also observed an abnormality in the cervical spine of 54 patients of $69 \mathrm{AD}$ patients who showed a neurological abnormality. It was observed that 21 patients of the $32 \mathrm{AD}$ patients in the damage of both the duodenum and the cervical spine. Results revealed that Methicillin-resistant Staphylococcus aureus (MRSA) was able to sterilize 25 cases out of all cases that were detected MRSA in this treatment from the patient's skin of AD and is reported in their Japanese Paper [17].

\section{Discussion}

Kobayashi, et al. reported that Staphylococcus aureus is involved in the onset and exacerbation of atopic dermatitis [5]. IgE antibody titers to the toxin which is produced by Staphylococcus aureus is able to reflect the pathogenesis of AD as shown in a number of reports [11-14]. Regarding the failure of organs other than the skin in patients with AD, Yamada, et al. reported colitis in patients with $\mathrm{AD}$, Kira, et al. reported cervical spine inflammation in patients with $\mathrm{AD}[7,9]$. Kino, et al. reported inflammation in the colon of infants with $\mathrm{AD}$ [8]. Treatment improved skin rash in these cases, as well as in the inflammation of the colon. Ito, et al. reported the neurologic findings and MRI of patients with $\mathrm{AD}$, they concluded that $\mathrm{AD}$ might become the risk factor in disc degeneration [10]. Results reported that the rate of disorder of the duodenum and the cervical spine in patients with AD is high.

The researchers developed a treatment method using a combination of disinfection therapy and Isodine ${ }^{\varpi}$ solution against Staphylococcus aureus, which causes skin rash, intestinal tract and cervical spine disorder in AD patients. Improvements were recorded regarding rash in many of the cases. Previously authors, reported duodenitis in 43 cases in duodenal biopsies of patients with $\mathrm{AD}$ of the 53 cases. Along with the improvement of the rash by Staphylococcal disinfection skin care method for AD that was developed, of 12 patients with repeated duodenum biopsies, the duodenitis was normalized in five cases [17]. Authors identified neurological abnormalities in 89 patients of $110 \mathrm{AD}$ patients. Using MRI, they had also observed an abnormality in the cervical spine of 54 patients of $69 \mathrm{AD}$ patients who showed a neurological abnormality. Authors had also observed 21 patients of the $32 \mathrm{AD}$ patients in the damage of both the duodenum and the cervical spine.

Results found that Staphylococcus aureus in the skin of patients with AD produces toxin at a high rate. Newborns with AD have skins infected with Staphylococcus aureus. In addition, research revealed that IgE antibodies to SEA and SEB were positive in the serum of patients with $\mathrm{AD}$, a few months after birth. In patients with $\mathrm{AD}$ there are many reports of correlation between changes in the severity of the transition and presence of IgE antibodies to SEA and SEB [11-14]. In cases where improvement of rash by our treatment was recorded, the number of cases of IgE antibodies to SEA and SEB value correlated with the total IgE value.

Ochi, et al. reported that superantigen is involved in disorders of the cervical spine. Already in the United States, bleach bath therapy is incorporated into the treatment guidelines of $\mathrm{AD}$, and the need for Staphylococcus aureus measures are coordinated to the treatment. The authors hope the treatment to minimize the effects of the toxins that Staphylococcus aureus is produced. In the treatment of $\mathrm{AD}$, the treatment method in which we have combined to common treatment methods of treatment to deal with the disinfection of Staphylococcus aureus that has developed is amazing in that regard.

\section{Conclusion}

Last year, authors had reported for the first time in the world that AD may be one of the Superantigens Diseases [21]. The Journal of Pharmaceutical Microbiology titled Staphylococcus aureus vs. AD published in April 2016 was published [22].

Since it has been demonstrated that Staphylococcus aureus in the onset or aggravation factors of AD are involved, in the future, we are sure the treatment that we have developed as a treatment to AD to be taken up by health practitioners. The treatment proposed (use of combined disinfectants) in this study alleviated skin rash in patients with AD. This treatment can also be chosen for the treatment of patients involved of MRSA as well as Staphylococcus aureus, which is detected from the skin of patients with atopic dermatitis. The authors hope that health practitioners would look into the proposed treatment method and adopt it.

\section{References}

1. Imayama S, Shimozono Y, Hoashi M, Yasumoto S, Ohta S, et al. (1994) Reduced secretion of immunogloblin-A to the skin surface of patients with atopic dermatitis. J Allergy Clin Immunol 94: 195-200.

2, Ternowitz T, Herlin T (1986) Defective monocyte and polymorphonuclear leukocyte chemotaxis and clinical characteristics in atopic dermatitis. Arch Dermatol Res 278: 454-9.

3. Schöpf E, Kapp A (1987) Chemiluminescence Response of Polymorphonuclear Leukocytes in Atopic Dermatitis. Int Arch Allergy Appl Immunonol 82: 380-2. 4. Hoeger PH, Niggemann B, Schroeder C (1992) Enhanced basal and stimulated PMN chemiluminescence activity in children with atopic dermatitis : stimulatory role of colonizing staphylococci ? Acta Pediatr 81: 6-7.

5, Kobayashi T, Glatz M, Horiuchi K, Kawasaki H, Akiyama H, et al. (2015) Dysbiosis and Staphylococcus aureus Colonization Drives Inflammation in Atopic Dermatitis. Immunity 42:756-66. 
6. Yamada H, Izutani R, Chihara J, Yudate T, Matsukura M, et al. (1996) RANTES mRNA expression in skin and colon of patients with atopic dermatitis. Int Arch Allergy Immunol 111(suppl 1): 19-21.

7, Kino M, Kojima T, Yamamoto A, Sasal M, Taniuchi S, et al. (2002) Bowel wall thickening in infants with food allergy. Pediatr Radiol 32: 31-3.

8. Kira J, Yamasaki K, Kawano Y, Kobayashi T (1997) Acute myelitis associated hyperIgEemia and atopic dermatitis. NeurolSci 148: $199-203$.

9. Ito S, Hattori T, Fukutake T, Sugimoto K (2003) Is atopic dermatitis a risk factor for intervertebral disc degeneration? A preliminary clinical and MRI study. J Neurol Sci 206: 39-42.

10. White J, Herman A, Pullen AM, Kubo R, K appler JW, Marrack P (1989) The Vbeta-specifi c superantigen staphylococcal enterotoxin B: stimulation of mature T cells and clonal detection in neonatal mice. Cell 56: 17-35.

11. Bunikowski R, Mielke M, Skarabis H, Herz U, Bergmann R, et al. (1998) Prevalence and role of serum IgE antibodies to the Staphylococcus aureus-derived superantigens SEA and SEB in children with atopic dermatitis. J Allergy Clin Immunol 103: 119-24.

12. Nomura I, Tanaka K, Tomita H, Katsunuma T, Ohya Y, et al. (1999) Evaluation of the staphylococcal exotoxins and their specific IgE in childhood atopic dermatitis. J Allergy Clin Immunol 104: 441-6.

13. Tada J, Toi Y, Akiyama H, Arata J, Kato H (1996) Presence of specific IgE antibodies to staphylococcal enterotoxins in patients with atopic dermatitis. Eur J Dermatol 6: 552-4.

14. Bunikowski R, Mielke MEA, Sakarabis H, Worm M, Anagnostopoulos I, et al. (2000) Evidence for a disease-promoting effect of Staphylococcus aureus -derived exotoxins in atopic dermatitis. J Allergy Clin Immunol 105: 814-9.

15. Sugimoto K, Ishikawa N, Terano T, Kitukawa Y, Kubosawa H, et al. (2006) The importance of Bacterial Superantigens Produced by Staphylococcus aureus in the Treatment of Atopic Dermatitis Using Povidone-Iodine. Dermatology 212: 26-34.

16. Sugimoto K, Kuroki H, Kanazawa M, Kurosaki T, Abe H, et al. (1997) New successful treatment with disinfectant for atopic dermatitis. Dermatol 195: 62-8. 17. Sugimoto K, Ishikawa N, Sugioka T, Nakamura M, Koneki H, et al. (1998) Isojin ${ }^{\circledR}$ disinfectant therapy for atopic dermatitis. Japanese J Pediatric Dermatol 17: $103-7$.

18. Sugimoto K, Ishikawa N, Ogawa A (1998) Treatment of Staphylococcus aureus by disinfection treatment of patients with atopic dermatitis. Hifu 40: 117-24.

19, Sugimoto K, Ishikawa N, Sugioka T, Koseki H, Kubosawa H, et al. (2002) The importance of disinfection therapy using povidone-iodine solution in atopic dermatitis. Dermatology 204: 63-9.

20. Ochi H, Osoegawa M, Murai H, Minohara M, Taniwaki T, et al. (2004) Presence of IgE Antibodies to Bacterial Superantigens and Increased IL-13-Producing T Cells in Myelitic Patients with Atopic Diathesis. Int Arch Allergy Immunol 134: 41-8.

21. Sugimoto K, Kitukawa Y, Aotsuka A, Wada T, Kubosawa H, et al. (2015) Is Atopic Dermatitis One of the Superantigens Diseases?. J Dermatolog Clin Res 3: 1052-3.

22, Sugimoto K (2016) Staphylococcus-aureus-vs-Atopic-Dermatitis. J Pharm Microbiol 2: 8.

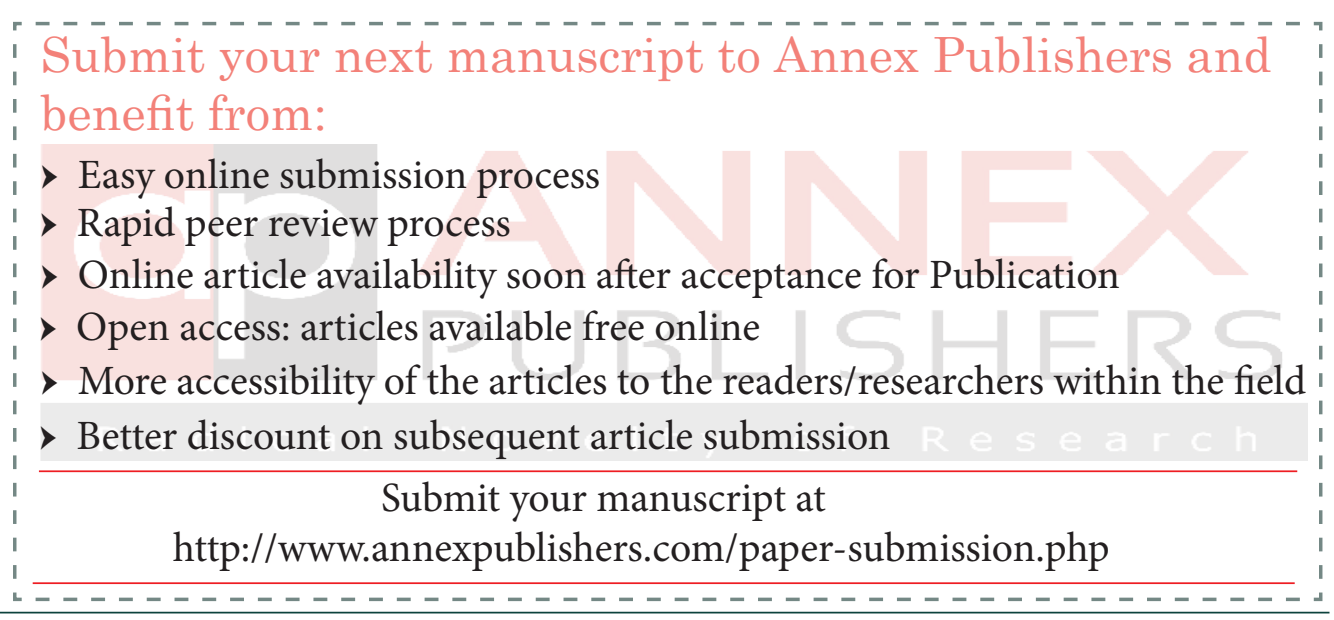

